\title{
The role of disease perceptions and results sharing in psychological adaptation after genetic susceptibility testing: the REVEAL Study
}

\begin{abstract}
Sato Ashida ${ }^{\star, 1}$, Laura M Koehly ${ }^{1}$, J Scott Roberts ${ }^{2}$, Clara A Chen ${ }^{3}$, Susan Hiraki ${ }^{4}$ and Robert C Green ${ }^{4,5,6}$
This study evaluates the extent to which psychological adaptation (validated measures of depressive symptoms, anxiety, and test-specific distress) after genetic susceptibility testing is influenced by changes in beliefs about Alzheimer's disease (AD) and sharing of test results with others. Adult children of $A D$ patients $(N=269)$ from a randomized clinical trial involving genetic testing for apolipoprotein $\mathrm{E}(A P O E)$ provided information before, as well as 6 weeks and 12 months after results disclosure. The levels of adaptation varied highly among participants at 12 -month assessment. Participants who learned that they were $\varepsilon 4$ negative (lower risk) had a reduction in perceived risk and concern about developing AD compared with those who learned that they were $\varepsilon 4$ positive. Those who received results through an extended educational protocol (three in-person visits) had a larger decline in $A D$ concern than those in a condensed protocol (educational brochure and two in-person visits). Increase in $A D$ concern 6 weeks after disclosure was associated with increase in depression scores $(b=0.20, P<0.01)$ and anxiety levels $(b=0.20, P<0.01)$, and higher distress associated with $A D$ genetic testing $(b=0.18, P=0.02) 1$ year after testing. Increase in perceived risk ( $b=0.16, P=0.04$ ) was also associated with higher $A D$ genetic testing distress. Sharing the test results with health professionals and friends (but not family) was associated with decrease in depression $(b=-0.11, P=0.05)$ and anxiety levels $(b=-0.16, P<0.01$ ), respectively after a year. Enhancing discussion with regard to risks and concerns about $A D$ during pretesting counseling and obtaining support through sharing the results after testing may help facilitate test recipients' longterm psychological adaptation.
\end{abstract}

European Journal of Human Genetics (2010) 18, 1296-1301; doi:10.1038/ejhg.2010.119; published online 28 July 2010

Keywords: susceptibility genetic testing; $A D ; A P O E$; results disclosure; communication; risk perceptions

\section{INTRODUCTION}

Genetic information is increasingly being used in our society to predict individuals' risk levels for disease, to target and tailor preventive health education, and to determine effective treatment strategies. Strongly predictive genetic tests for such conditions as Huntington's disease (HD), hereditary breast and ovarian cancer (HBOC), and hereditary nonpolyposis colorectal cancer (HNPCC) have been used in health-care settings for decades. Although predictive testing for HD provides information on whether a person carries the disease causing mutation, testing for hereditary cancer syndromes have lower predictive values (eg, $70-82 \%$ for $\mathrm{HBOC}^{1}$ and $47-85 \%$ for HNPCC $^{2}$ ). Genetic susceptibility tests for common complex diseases that have become available more recently have even lower predictive values and provide information on only a modest increase in disease risks. These susceptibility tests are currently considered to have no or little clinical utility, ${ }^{3}$ and studies looking at their psychological impacts are limited. However, some of these tests are already available to the public through direct-to-consumer companies ${ }^{4}$ even for conditions for which effective treatments are not available (eg, Alzheimer's disease $(\mathrm{AD})$ ), underscoring the importance of understanding their impacts on those who choose to undergo such testing.
There is a substantial literature exploring the impact of genetic testing and disclosure of highly penetrant conditions. A review of literature showed that individuals undergoing predictive genetic testing (eg, HD, HBOC, and familial adenomatous polyposis) generally do not show psychological distress. ${ }^{5}$ However, psychological distress among some recipients of genetic susceptibility testing for HNPCC has been reported. ${ }^{6,7}$ In a review, Meiser ${ }^{8}$ observed differences in the psychological impacts of genetic test results between HNPCC and HBOC, and noted that the impacts may differ depending on the characteristics of the disease (eg, controllability, availability of preventive strategies). As the predictive ability of genetic susceptibility tests for common diseases is much lower compared with the predictive tests, results are likely to have different impacts on individuals.

In all, $30-50 \%$ of $\mathrm{AD}$ population risk is attributed to genetics. ${ }^{9,10}$ Individuals who carry mutations in APP, PS1, and PS2 genes ( $<2 \%$ of $\mathrm{AD}$ cases) are almost certain to develop the hereditary form of $\mathrm{AD} .{ }^{11}$ On the other hand, late-onset AD ( $>98 \%$ of cases) usually penetrates after the age of 65 years, and has been linked to a susceptibility gene, apolipoprotein $\mathrm{E}(\mathrm{APOE}){ }^{9}$ Having one or two $\varepsilon 4$ alleles (APOE $\varepsilon 4+$ ) is associated with increased risk of $\mathrm{AD}$ (3 times and 8-30 times the population risk, respectively). ${ }^{9}$ Use of this test for diagnostic or

${ }^{1}$ Social and Behavioral Research Branch, National Human Genome Research Institute, Bethesda, MD, USA; ${ }^{2}$ Department of Health Behavior and Health Education, University of Michigan School of Public Health, Ann Arbor, MI, USA; ${ }^{3}$ Data Coordinating Center, Boston University School of Public Health, Boston, MA, USA; ${ }^{4}$ Department of Neurology, Boston University School of Medicine, Boston, MA, USA; ${ }^{5}$ Department of Epidemiology, Boston University School of Public Health, Boston, MA, USA and ${ }^{6}$ Department of Medicine (Genetics), Boston University School of Medicine, Boston, MA, USA

${ }^{*}$ Correspondence: Dr S Ashida, Social and Behavioral Research Branch, National Human Genome Research Institute, 31 Center Drive, B1B37C, Bethesda, MD 20892-2073, USA. Tel: +301 443 1610; Fax: +301 480 3108; E-mail: ashidas@mail.nih.gov

Received 3 December 2009; revised 18 May 2010; accepted 9 June 2010; published online 28 July 2010 
predictive purposes is not recommended in clinical practice because of the limited predictive values of testing and lack of preventive strategies. Yet, surveys suggest that many people at risk for $\mathrm{AD}$ are interested in testing. ${ }^{12}$

Recognizing the importance of understanding the impacts of genetic susceptibility tests that are already available to the public, a series of clinical trials have been conducted to investigate the safety and feasibility of providing $A P O E$ test results to first-degree relatives of AD patients. ${ }^{13}$ Despite the limited clinical utility, the APOE testing exhibits good analytic and clinical validity, providing a context in which the impacts of modestly predictive genetic susceptibility information can be examined. The results from the first trial showed that in a carefully screened population of volunteers and with the support of genetic counselors, there was no serious psychological distress associated with provision of $A P O E$ test results. ${ }^{13}$ However, the results from the first trial also showed individual differences in psychological responses, ${ }^{14}$ with some participants experiencing transient distress after result disclosure.

Cognitive responses to disease information determine how individuals cope with stressful health-risk information and ultimately influence the outcomes of coping processes. ${ }^{15}$ Previous studies concerning strongly predictive genetic tests showed that individuals' illness beliefs or cognitions may change after receiving genetic testing, ${ }^{16,17}$ and such illness beliefs were associated with post-test psychological distress. ${ }^{18}$ It is possible that beliefs about the illness also change after receiving modestly predictive genetic susceptibility test results, and the extent to which these beliefs change may determine how test recipients cope and adapt.

Communication of test results to family has been reported to be problematic among the recipients of HD-predictive test, ${ }^{19,20}$ and can lead to diminished support ${ }^{21}$ or strained relationships within families. ${ }^{17}$ If test results are communicated outside the family, there is also a potential for insurance or employment discrimination and stigmatization. ${ }^{12,22}$ On the other hand, communication of the test results with regard to a lower penetrance condition for which preventive strategies are available, HNPCC, has been reported to increase family support, ${ }^{23}$ leading to better psychological adjustment among test recipients. ${ }^{24}$ Although effective preventive and treatment strategies are not available for late-onset $\mathrm{AD}$, predictive ability of the $\mathrm{AD}$-susceptibility test is considerably lower compared with HD. It may be that recipients of $\mathrm{AD}$-susceptibility testing have less difficulty in communicating the results to others and that such behavior helps them obtain support to facilitate psychological well-being.

This study aims to investigate how changes in AD-related beliefs (ie, perceived risk, concerns about developing $\mathrm{AD}$, and causal beliefs) and communication of test results to others soon after receiving APOE genetic test results (6 weeks) affect long-term changes ( 12 months) in psychological well-being. It is hypothesized that an increase in risk perceptions (perceived risk, $\mathrm{AD}$ concern) and a decrease in perceived control (increase in genetics/heredity and decrease in lifestyle causal attribution) would be associated with an increase in psychological distress, and communication of the test results would be associated with a decrease in distress.

\section{MATERIALS AND METHODS}

\section{Participants and procedure}

The REVEAL Study is a series of randomized controlled trials that evaluate the feasibility, safety, and psychological impacts and behavioral outcomes of APOE genetic testing among unaffected adults with one first-degree relative affected by $\mathrm{AD}$ (developed after the age of 60 years). ${ }^{13}$ This paper considers participants of the REVEAL II Study (data collected from February 2003 to May 2005) who
Table 1 Estimated lifetime risk of developing AD provided to participants and frequency of each genotype

\begin{tabular}{lllll}
\hline & \multicolumn{2}{c}{ White } & \multicolumn{2}{c}{ African American } \\
Genotype & Males & Females & Males & Females \\
\hline$\varepsilon 2 / \varepsilon 3$ & $13 \%(6)$ & $19 \%(10)$ & $33 \%(0)$ & $36 \%(7)^{\mathrm{a}}$ \\
$\varepsilon 3 / \varepsilon 3$ & $18 \%(35)$ & $29 \%(75)$ & $41 \%(4)$ & $49 \%(22)$ \\
$\varepsilon 2 / \varepsilon 4$ & $25 \%(5)$ & $49 \%(4)$ & $48 \%(0)$ & $69 \%(2)$ \\
$\varepsilon 3 / \varepsilon 4$ & $29 \%(24)$ & $52 \%(47)$ & $56 \%(2)$ & $73 \%(13)$ \\
$\varepsilon 4 / \varepsilon 4$ & $56 \%(3)$ & $57 \%(8)$ & $77 \%(0)$ & $74 \%(2)$
\end{tabular}

ancludes one participant who had $\varepsilon 2 / \varepsilon 2$ genotype and was informed that genotype-specific estimates could not be calculated because of a small number of individuals with this genotype in the population.

Notes: Numbers in parentheses indicate numbers of participants who received relevant risk estimates.

received $A P O E$ genetic testing and risk assessment. All participants were English speaking and individuals exhibiting cognitive impairment or clinically significant depression or anxiety were not eligible.

Participants received $A P O E$ test results and estimated lifetime risk of disease based on age, gender, race, and $A P O E$ genotype (see Table 1) in either an extended (in-person education, individual counseling, neuropsychological, and psychological screening, and blood draw; mean length of in-person counseling $=76 \mathrm{~min}$ ) or a condensed (educational brochure by mail, question and answer session, neuropsychological and psychological screening, and blood draw; mean length of in-person counseling=33 min) protocol with a genetic counselor or a physician. During the in-person disclosure session, participants were presented with graphs representing $\mathrm{AD}$ risks for the general population, first-degree relatives of $\mathrm{AD}$ patients, and those with specific genotypes, and were informed about the limitations of these estimates. Detailed information with regard to the development of risk estimates ${ }^{25}$ and genetic counseling procedures $^{26}$ are explained elsewhere.

After baseline assessment (AD-related beliefs, depressive symptoms, anxiety, and demographic characteristics), follow-up assessments occurred at 6 weeks (AD-related beliefs and communication of test results), and 1 year after disclosure (depressive symptoms, anxiety, and impact of genetic testing). Out of 411 individuals who showed interest in the study and met eligibility criteria, 280 (68\%) received genotype-based risk assessment. Participants who provided information at all assessment points and those who identified themselves as white or African American were included in this report $(N=269)$. Four participants in other racial categories were excluded because of a limited ability to examine their unique reactions to genetic risk information with available data.

\section{Measures}

The Center for Epidemiology Studies-Depression Scale (CES-D) ${ }^{27}$ was used, and participants who scored higher than the clinical cut point of 16 at baseline were given increased monitoring during the study. Those who scored $>27$ were excluded. Anxiety symptoms were assessed using the well-validated Beck Anxiety Inventory (BAI). ${ }^{28,29}$ The scores range between 0 and 63 with higher scores reflecting more anxiety. The Impact of Genetic Testing for Alzheimer's disease (IGT-AD) was developed based on a well-established measure, the Multidimensional Impact of Cancer Risk Assessment Questionnaire. ${ }^{30}$ This situation-specific IGT-AD was used to measure the psychological impacts of $\mathrm{AD}$ genetic susceptibility testing and risk assessment that may not have been captured by the general distress measures (eg, CES-D and BAI). The distress subscale of the IGT-AD (12 items, see Appendix A) showed good validity and reliability. ${ }^{31}$ The scores range between 0 and 60 with higher scores reflecting higher levels of distress.

For perceived risk, participants rated belief about their own chances of developing $\mathrm{AD}$ during their lifetime on a scale of $0-100 \%$. AD concern was measured using five items adopted from a previous study. ${ }^{32}$ Three items assessed the extent to which participants were concerned that they would develop $\mathrm{AD}$, that they would develop it in the next 5 years or at some point later in their lives ( $1=$ 'strongly disagree' to $5=$ 'strongly agree'). Two items 
asked about their belief that they would someday develop $\mathrm{AD}$ and the extent to which participants felt that $\mathrm{AD}$ was the worst disease of which they could think. An average of five items was calculated (Cronbach $\alpha=0.67$ and 0.60 for baseline and at 6 weeks, respectively). Two items assessed participants' causal beliefs and were considered individually in the analyses (How important is genetics/ heredity or lifestyle in increasing risk of $\mathrm{AD}$ ?: $1=$ 'not important', $3=$ 'somewhat important', and $5=$ 'very important'). ${ }^{32}$ At the 6-week assessment, participants were asked 'Have you told anyone the results of your APOE genetic test?' followed by a group of questions, 'Who did you tell about the results of your $A P O E$ genetic test: family member, spouse/significant other, friends, health professional?'

Age, years of education, gender, race, marital status, presence of long-term care insurance, and past caregiving experience were assessed through selfreport. Indicator variables for age ( $\geq 60$ years), years of education ( $\geq 16$ years), race (white), insurance status (have long-term care insurance), and caregiving experience (ever provided care to individual(s) with $\mathrm{AD}$ ) were created. Genotype status was coded to indicate whether a participant was found to carry at least one $A P O E \& 4$ allele (positive).

\section{Analysis}

Three multiple regression models (CES-D, BAI, and IGT-AD distress) were fitted using SPSS. The demographic variables were evaluated as potential covariates, and those that were significantly associated with changes in one or more psychological outcomes (race and marital status), participants' genotype, and randomization status (extended $v s$ condensed disclosure protocol) were controlled in each model. The models for CES-D and BAI also controlled for the baseline measures. None of the participants previously experienced genetic testing for $\mathrm{AD}$ and their baseline levels were assumed to be equal in the IGT-AD model.

Four AD-belief variables (perceived risk, AD concern, causal beliefs for genetics/heredity, and lifestyle factors) were entered in a forward stepwise selection manner controlling for covariates, treating baseline and 6-week change scores as a pair to assess the significance of the changes while controlling for the respective baseline values. Thus, the baseline score was included in the model regardless of its significance if the relevant change score was significant. Significant baseline belief variables were retained even if respective change scores were not significant and removed from the model as this indicates a relative importance of an initial belief compared with change in this belief after receiving the test results. Communication variables were also entered in a forward stepwise selection manner with covariates and significant belief variables. Statistical significance was determined based on a Type I error rate of 0.05 , and standardized coefficients are presented in results.

\section{RESULTS}

Demographic characteristics of the participants are shown in Table 2. The average age of the participants was 58.1 years $(\mathrm{SD}=10.6)$ ranging from 33 to 86 , and the average years of education obtained was 16.1 years $(\mathrm{SD}=2.6)$ ranging from 3 to 20 years. The average residual lifetime risk estimate provided to participants was $32.6 \%(\mathrm{SD}=15.86$,

Table 2 Demographic characteristics of the sample $(N=269)$

\begin{tabular}{lc}
\hline & Yes (\%) \\
\hline Age $\geq 60$ years & 41.6 \\
Education: $\geq 16$ years & 68.4 \\
Male & 29.4 \\
White & 80.7 \\
Married & 61.3 \\
Currently have long-term care insurance & 21.6 \\
Carrier of $\varepsilon 4$ allele & 40.9 \\
Have current or past caregiving experience & 36.1 \\
Extended disclosure protocol & 33.3 \\
\hline
\end{tabular}

ranging from 3 to $72 \%$ ): it was not correlated with participants' baseline perceived risk $(r=0.04, P=0.54)$ but correlated with their 6-week reports of perceived risk $(r=0.41, P<0.01)$.

Table 3 presents the descriptive statistics of the psychological outcome variables. The change in CES-D between baseline and 12month assessments ranged from -24 to 14 , with $45 \%$ reporting an increase in depressive symptoms and 55\% reporting no change or decrease. Twenty-four participants (9\%) reported scores higher than the clinical cut point of 16 at 12-month follow-up, and 21 of these 24 individuals had scores below the cutoff at baseline. The change in BAI score ranged from -18 and 22 , with $33 \%$ reporting an increase. The mean IGT-AD distress score at 12 -month assessment was 1.32 , ranging from 0 to 17.

Descriptive statistics for the $\mathrm{AD}$-belief and communication variables are shown in Table 4 . The average change in perceived risk of developing $\mathrm{AD}$ decreased significantly more among those who received negative $(M=-11.61 \%)$ than those who received positive results $(M=-0.94 \%, t=-3.28, P<0.001)$. Those who received negative results also exhibited a significantly larger decrease in $\mathrm{AD}$ concern $(M=-0.39)$ at 6 weeks than those who received positive results $(M=-0.05, t=-3.85, P<0.001)$; those who were in an extended disclosure condition exhibited a significantly larger decline in concern $(M=-0.38)$ compared with those who were in a condensed condition $(M=-0.19, t=2.04, P=0.04)$. Large proportions of the participants shared their results with family members $(62 \%)$ and spouse or significant others at 6 weeks $(52$, or $70 \%$ of those with a spouse/ partner). In total, 35 and $15 \%$ shared with friends and health professionals, respectively.

Table 3 Descriptive statistics for the outcome variables

\begin{tabular}{lcccc}
\hline & $N$ & Mean (SD) & Minimum & Maximum \\
\hline CES-D: baseline & 268 & $5.56(5.05)$ & 0.00 & 26.00 \\
CES-D: at 12 months & & $6.25(6.11)$ & 0.00 & 29.00 \\
BAI: baseline & 267 & $3.34(4.08)$ & 0.00 & 24.00 \\
BAI: at 12 months & & $3.54(4.67)$ & 0.00 & 28.00 \\
IGT-AD distress: at 12 months & \multirow{2}{*}{269} & $1.34(2.76)$ & 0.00 & 17.00 \\
\hline
\end{tabular}

Table 4 Descriptive statistics for the independent variables ( $N=269)$

\begin{tabular}{|c|c|c|c|}
\hline & Mean (SD) & Minimum & Maximum \\
\hline \multicolumn{4}{|l|}{ AD beliefs at baseline } \\
\hline Perceived risk & $50.97(22.43)$ & 0.00 & 100.00 \\
\hline Perceived risk (change) ${ }^{a}$ & $-7.21(26.50)$ & -70.00 & 100.00 \\
\hline$A D$ concern & $3.40(0.69)$ & 1.60 & 5.00 \\
\hline$A D$ concern (change) ${ }^{a}$ & $-0.25(0.71)$ & -2.40 & 1.80 \\
\hline Causal belief: genetic & $4.07(0.85)$ & 1.00 & 5.00 \\
\hline Causal belief: genetic (change) & $0.09(0.86)$ & -2.00 & 3.00 \\
\hline Causal belief: lifestyle & $3.49(1.11)$ & 1.00 & 5.00 \\
\hline Causal belief: lifestyle (change) ${ }^{a}$ & $-0.09(1.11)$ & -3.00 & 3.00 \\
\hline Told GT result to ${ }^{\mathrm{b}}$ & Yes (\%) & & \\
\hline Family member & $167(62.1)$ & - & - \\
\hline Spouse/significant other & $140(52.0)$ & - & - \\
\hline Friend & $94(37.5)$ & - & - \\
\hline Health professional & $40(14.9)$ & - & - \\
\hline
\end{tabular}

${ }^{a}$ Change scores indicate changes between baseline and 6-week assessment.

${ }^{b}$ Communication data based on 268 individuals. 
Table 5 Regression coefficients of factors predicting psychological states at 12 months

\begin{tabular}{|c|c|c|c|}
\hline Variables & $C E S-D(N=268)$ & $B A I(N=267)$ & IGT-AD distress $(N=269)$ \\
\hline Perceived risk & $0.22^{* * *}$ & - & 0.10 \\
\hline$A D$ concern & 0.02 & 0.12 & $0.19 *$ \\
\hline AD concern (change) & $0.20 * *$ & $0.20 * *$ & $0.18^{*}$ \\
\hline Causal belief: genetic & $-0.16^{* *}$ & - & - \\
\hline Told friend & - & $-0.17^{* *}$ & - \\
\hline Told healthcare professional & $-0.10^{*}$ & - & - \\
\hline
\end{tabular}

${ }^{*} P<0.05 ;{ }^{* *} P<0.01 ; * * * P<0.001$.

Each column represents an individual model for each outcome.

Each model is controlled for race, marital status, genotype, and respective psychological control measure (baseline for
AD-belief and communication variables that were not significant in any of the models are not presented in this table.

Race (White) was significant in CES-D $(b=0.133, P=0.018)$ and BAI $(b=0.128, P=0.029)$, and $\varepsilon 4$ carrier was significant in IGT-AD $(b=0.137, P=0.032)$ model.

The final models are presented in Table 5. An increase in CES-D at the 12-month assessment was associated with higher levels of perceived risk $(b=0.22, P<0.01)$ and lower levels of causal attribution to genetics/heredity $(b=-0.16, P=0.01)$ at baseline, and a short-term increase (6 weeks after the disclosure) in $\mathrm{AD}$ concern $(b=0.20$, $P<0.01)$. Sharing APOE test results with health professionals was associated with a decrease in CES-D at the 12-month assessment $(b=-0.10, P=0.05)$. A short-term increase in $\mathrm{AD}$ concern was associated with an increase $(b=0.20, P<0.01)$ and sharing the results with friends was associated with a decrease $(b=-0.17, P<0.01)$ in BAI. Baseline $\mathrm{AD}$ concern $(b=0.19, P=0.04)$ and an increase in concern at 6 weeks $(b=0.18, P=0.02)$, along with a short-term increase in perceived risk for $\mathrm{AD}(b=0.17, P=0.04)$, were associated with higher levels of the IGT-AD distress at 12 months.

\section{DISCUSSION}

There were individual differences in the directions and extent to which long-term changes occurred in general psychological (depressive symptoms, anxiety levels) measures and in the levels of situation-specific distress (impact of $\mathrm{AD}$ genetic testing) among the participants in this study after they received $A P O E$ test results. In all, $9 \%$ of the participants who had low levels of depression at baseline reported clinically significant levels 12 months later. Although not clinically significant and it may be attributed to factors other than genetic testing, ${ }^{33}$ some additional participants showed an increase in general distress measures (CES-D and BAI) 12 months after receiving the test results.

Participants in this study also reported a wide range of perceived risk for developing $\mathrm{AD}$ before undergoing genetic testing. Reported changes in risk perceptions were not only very wide in range but also in different directions (ranging from a decrease of $75 \%$ to an increase of $100 \%$ ) suggesting individual differences in reactions to the genetic susceptibility risk information. Similar to a previous report, ${ }^{34}$ risk assessment on the basis of $A P O E$ genotype influences disease risk perceptions, as participants in the current study who were identified not to carry the risk version showed more decrease in perceived risk and $\mathrm{AD}$ concern compared with those who were identified to carry an $\varepsilon 4$ allele. Changes in perceived causes of $\mathrm{AD}$ were not observed in the current study possibly because of participants believing in the importance of genetics/heredity at baseline as all participants in this study chose to undergo APOE testing, leaving little potential for changes to occur at the 6-week assessment.

Our results suggest that changes in beliefs and perceptions about the disease after the receipt of genetic susceptibility test results may influence how test recipients psychologically adapt over time. An increase in concern about developing $\mathrm{AD}$ shortly after receiving the test results was associated with an increase in depressive symptoms and anxiety levels, and higher distress associated with $\mathrm{AD}$ genetic testing 12 months later. Efforts to decrease $\mathrm{AD}$ concerns during the disclosure session (eg, reiterating the limitations of current risk assessment, using gain/loss framing of information, and highlighting research advances in the development of $\mathrm{AD}$ treatment), or tailoring resources to address specific concerns raised by test recipients may be beneficial in facilitating their long-term psychological adjustment. As 84 -positive individuals reported more increase in $\mathrm{AD}$ concern compared with those who were $\varepsilon 4$ negative, the former group may especially benefit from additional support and intervention. Participants who were in the extended disclosure condition reported a significant decrease in $\mathrm{AD}$ concern, and hence, this may justify the provision of face-to-face pretesting genetic education.

Individuals who hold high levels of perceived risk before AD-susceptibility testing and those who report increase in this perception shortly after results disclosure may experience increase in depression or higher distress associated with APOE testing. An average perceived risk reported by participants at baseline was about $50 \%$, whereas the average risk estimates given to these participants was $32 \%$. Almost $80 \%$ of the participants reported higher perceived risk than the risk estimate they received, with $50 \%$ overestimating by $\geq 20 \%$. Although the perceived risk at the 6-week assessment became closer to the objective risk estimates provided to participants after testing compared with the perception at baseline, the correlation was only moderate $(r=0.41)$. Assessing perceived risk before and after individuals undergo genetic susceptibility testing may allow the identification of those who may benefit from additional interventions to move their perceptions toward levels closer to their objective estimated risk. As those who received positive results reported less decrease in risk perception, those who report high-risk perception at baseline and receive positive results can be targeted. Participants who believed genetics/heredity as important causes of $\mathrm{AD}$ at baseline to a lesser extent experienced increased depressive symptoms at 12 months. It may be that individuals with less knowledge about the role of genetics were affected more by the new information provided during the study than those who had higher awareness. Research on $\mathrm{AD}$ is progressing rapidly, and new potential preventive factors are being identified and evaluated. ${ }^{35}$ Providing up-to-date scientific knowledge may help prevent potential psychological consequences of providing genetic susceptibility test results. 
As hypothesized, talking to a health-care professional about the $A P O E$ test result was associated with a decrease in depressive symptoms, and talking to friends was associated with a decrease in anxiety levels over a year following testing. Learning about the condition or obtaining support within social networks can help individuals adapt to threatening personal health events. ${ }^{36}$ Sharing test results may have allowed some participants to obtain additional information or social and medical support to facilitate their general adaptation. However, it is important to consider potential negative aspects associated with sharing results with nonfamily members such as limitations in obtaining insurance or employment, ${ }^{22}$ as recipients of predictive testing for HD have expressed such concerns. ${ }^{37}$ As predictive value of the APOE testing is much lower compared with the test for HD, recipients of $A P O E$ testing may face such problems to a lesser extent. However, it may be helpful for genetic counselors to discuss both benefits and consequences of sharing test results during clinical contacts to help individuals make informed decisions. Given that legal protections against genetic discrimination do not cover the longterm care insurance industry, and our previous studies have suggested that individuals use genetic testing to inform insurance purchasing behavior, ${ }^{38,39}$ this domain in particular may warrant discussion. Further studies investigating the content of communication are needed to understand the mechanisms through which interpersonal interactions may influence long-term psychological adjustment.

Communication to family and/or spouse was not significantly associated with psychological outcomes in this study. This may partly be due to low variability in responses among the participants $(79 \%$ communicated). However, it may also be because disclosing genetic risk information to family members has positive and negative consequences that are different from disclosing to nonfamily members. Disclosing test results allows individuals to inform their family members of their risks and encourage preventive behaviors if available. ${ }^{19,40,41}$ At the same time, it may cause distress among family members if the condition is threatening, highly penetrant, and/or no effective preventive strategies are available (eg, HD)..$^{20,42}$ Like HD, lateonset $\mathrm{AD}$ is a threatening neurodegenerative disorder without effective preventive strategies. However, the penetrance of $\mathrm{AD}$ in persons who are $\varepsilon 4$ positive is considerably lower and this may explain the nonsignificant impact of sharing results with family members in this study. Future studies would benefit by distinguishing different types of family members (eg, biological and nonbiological kin) to help decipher differential reasons for communication as well as distinct benefits and consequences of results sharing that may influence psychological adaptation of genetic susceptibility test recipients.

A majority of participants in this study were highly educated, limiting the generalizability of the results to a larger population. However, this study included $20 \%$ African-American participants, which is notable because of the general underrepresentation of this population in the field of genetic research. Assessments in this study were conducted through self-report. The reliability of one of the variables, $\mathrm{AD}$ concern, was rather low $(r=0.67$ and 0.60$)$, potentially influencing its observed importance in the models. The results of this study should be interpreted while considering these limitations.

\section{CONCLUSIONS}

As the use of genetic susceptibility testing for common diseases increases, preventing unwanted psychological impacts of genetic information becomes important. This is especially important when tests are already available to the public and address conditions such as $\mathrm{AD}$ for which no effective preventive or treatment strategies are available. Such genetic test results need to be provided with appropriate interventions to minimize negative impacts. The findings of this study suggest the benefits of assessing individuals' illness beliefs such as perceived risk and concern about developing the disease before and after providing a genetic-testing service, and developing tailored interventions that address these beliefs to facilitate psychological wellbeing of test recipients. Further studies are needed to identify other potential ways to prevent psychological distress among genetic susceptibility test recipients. This study showed that sharing test results with others may help facilitate psychological adaptation. Further research to investigate the potential mechanisms of these associations (eg, obtaining informational and emotional support) would be needed to inform future interventions.

\section{CONFLICT OF INTEREST}

The authors declare no conflict of interest.

\section{ACKNOWLEDGEMENTS}

The REVEAL Study was funded by the ELSI Branch of the National Human Genome Research Institute and the National Institute on Aging (R01 HG/ AG02213 and R01 AG09029). Additional support was provided by an NIA Mentoring Award to Dr Green (K24 AG027841), the Boston University Alzheimer's Disease Center (P50 AG13846) and Boston University General Clinical Research Center (GCRC) (M01 RR00533). The completion of this article was supported by the Intramural Research Program of the National Human Genome Research Institute at the National Institutes of Health. Other REVEAL investigators include: Lindsay A Farrer, PhD, Department of Neurology and Medicine (Genetics Program), Boston University School of Medicine and Biostatistics, Boston University School of Public Health; Robert Stern, PhD, Department of Neurology, Boston University School of Medicine; L Adrienne Cupples, PhD, Department of Epidemiology and Biostatistics, Boston University School of Public Health; Anil Nair, MD, Department of Neurology, Boston University School of Medicine; Erin Linnenbringer, MS, CGC, Department of Health Behavior and Health Education, University of Michigan School of Public Health; Thomas Obisesan, MD, MPH, Department of Medicine, Howard University Hospital, Washington, DC; Grace-Ann Fasaye, ScM, CGC, Department of Medicine, Howard University Hospital, Washington, DC; Charmaine Royal, PhD, National Human Genome Center, Howard University, Washington, DC; Melissa Barber, ScM, Memory and Aging Center, Case Western Reserve University/University Hospitals of Cleveland Memory and Aging, Cleveland, OH; Peter Whitehouse, MD, Memory and Aging Center, Case Western Reserve University/University Hospitals of Cleveland Memory and Aging, Cleveland, OH; Normal Relkin, MD, PhD, Department of Neurology, Weill Medical College of Cornell University, New York, NY; Elana Cox, MS, CGC, Department of Neurology, Weill Medical College of Cornell University, New York, NY; Lisa Ravdin, PhD, Department of Neurology and Neuroscience, Weill Medical College of Cornell University, New York, NY; Robert Cook-Deegan at Duke University.

\section{DISCLAIMER}

The views expressed in this article are those of the authors and do not necessarily reflect the official policy or position of the Department of Health and Human Services, nor the US Government.

1 Easton D: Breast cancer genes - what are the real risks? Nat Genet 1997; 16: 210-211.

2 Jarvinen $\mathrm{HJ}$, Aarnio $\mathrm{M}$, Mustonen $\mathrm{H}$ et al: Controlled 15-year trial on screening for colorectal cancer in families with hereditary nonpolyposis colorectal cancer. Gastroenterol 2000; 118: 829-834.

3 Feero WG, Guttmacher AE, Collins FS: The genome gets personal - almost. JAMA 2008; 299: 1351-1352.

4 Offit K: Genomic profiles for disease risk: predictive or premature? JAMA 2008; 299: $1353-1355$.

5 Lerman C, Croyle RT, Tercyak KP, Hamann H: Genetic testing: psychological aspects and implications. J Consult Clin Psychol 2002; 70: 784-797. 
6 Gritz ER, Peterson SK, Vernon SW et al: Psychological impact of genetic testing for hereditary nonpolyposis colorectal cancer. J Clin Oncol 2005; 23: 1902-1910.

7 Shiloh S, Koehly L, Jenkins J, Martin J, Hadley D: Monitoring coping style moderates emotional reactions to genetic testing for hereditary nonpolyposis colorectal cancer: a longitudinal study. Psychooncology 2008; 17: 746-755.

8 Meiser B: Psychological impact of genetic testing for cancer susceptibility: an update of the literature. Psychooncology 2005; 14: 1060-1074.

9 Farrer LA, Cupples LA, Haines JL et al: Effects of age, sex, and ethnicity on the association between apolipoprotein E genotype and Alzheimer disease. A meta-analysis. APOE and Alzheimer Disease Meta Analysis Consortium. JAMA 1997; 278: 1349-1356.

10 Silverman JM, Li G, Zaccario ML et al: Patterns of risk in first-degree relatives of patients with Alzheimer's disease. Arch Gen Psychiatry 1994; 51: 577-586.

11 McConnell LM, Koenig BA, Greely HT, Raffin TA: Genetic testing and Alzheime disease: recommendations of the Stanford Program in Genomics, Ethics, and Society. Genet Testing 1999; 3: 3-12.

12 Neumann PJ, Hammitt JK, Mueller C et al: Public attitudes about genetic testing for Alzheimer's disease. Health Aff 2001; 20: 252-264.

13 Green RC, Roberts JS, Cupples LA et al: Disclosure of APOE genotype for risk of Alzheimer's disease. New Engl J Med 2009; 361: 245-254.

14 Gooding HC, Organista K, Burack J, Biesecker BB: Genetic susceptibility testing from a stress and coping perspective. Soc Sci Med 2006; 62: 1880-1890.

15 Leventhal H, Benyamini Y, Brownlee S, Diefenbach MA, Leventhal EA, Patrick-Miller L: IIIness representations: theoretical foundations; in: Petrie KJ, Weinman JA (eds): Perceptions of Health and IIIness: Current Research and Applications. Singapore: Harwood Academic Publishers, 1997. pp. 19-45.

16 Claes E, Evers-Kiebooms G, Denayer L et al: Predictive genetic testing for hereditary breast and ovarian cancer: psychological distress and illness representations 1 year following disclosure. J Genet Counseling 2005; 14: 349-363.

17 Williams JK, Schutte DL, Evers C, Holkup PA: Redefinition: coping with normal results from predictive gene testing for neurodegenerative disorders. Res Nurs Health 2000; 23: 260-269.

18 van Oostrom I, Meijers-Heijboer H, Duivenvoorden HJ et al: Prognostic factors for hereditary cancer distress six months after BRCA1/2 or HNPCC genetic susceptibility testing. Eur J Cancer 2007; 43: 71-77.

19 Forrest K, Simpson SA, Wilson BJ et al: To tell or not to tell: barriers and facilitators in family communication about genetic risk. Clin Genet 2003; 64: 317-326.

20 Hamilton RJ, Bowers BJ, Williams JK: Disclosing genetic test results to family members. J Nurs Scholarsh 2005; 37: 18-24.

21 Sobel S, Cowan CB: Ambiguous loss and disenfranchised grief: the impact of DNA predictive testing on the family as a system. Fam Process 2003; 42: 47-57.

22 Clayton EW: Ethical, legal, and social implications of genomic medicine. N Engl J Med 2003; 349: 562-569.

23 van Oostrom I, Meijers-Heijboer H, Duivenvoorden $\mathrm{HJ}$ et al: A prospective study of the impact of genetic susceptibility testing for BRCA1/2 or HNPCC on family relationships. Psychooncology 2007; 16: 320-328.

24 van Oostrom I, Meijers-Heijboer H, Duivenvoorden HJ et al: Family system characteristics and psychological adjustment to cancer susceptibility genetic testing: a prospective study. Clin Genet 2007; 71: 35-42.
25 Cupples LA, Farrer LA, Sadovnick AD, Relkin N, Whitehouse P, Green RC: Estimating risk curves for first-degree relatives of patients with Alzheimer's disease: the REVEAL study. Genet Med 2004; 6: 192.

26 Linnenbringer E, Roberts JS, Hiraki S, Cupples LA, Green RC: 'I know what you told me, but this is what I think:' perceived risk of Alzheimer disease among individuals who accurately recall their genetics-based risk estimate. Genet Med 2010; 12: 219-227.

27 Radloff LS: The CES-D scale: a self-report depression scale for research in the general population. App/ Psych Meas 1977; 1: 385-401.

28 Beck AT, Epstein N, Brown G, Steer RA: An inventory for measuring clinical anxiety: psychometric properties. J Consult Clin Psych 1988; 56: 893-897.

29 Wetherell JL, Arean PA: Psychometric evaluation of the Beck Anxiety Inventory with older medical patients. Psychol Assess 1997; 9: 136-144.

30 Cella D, Hughes C, Peterman A et al: A brief assessment of concerns associated with genetic testing for cancer: the Multidimensional Impact of Cancer Risk Assessment (MICRA) questionnaire. Health Psychol 2002; 21: 564-572.

31 Chung WW, Chen CA, Cupples LA et al: A new scale measuring psychological impact of genetic susceptibility testing for Alzheimer's disease. Alz Dis Assoc Dis 2009; 23: 50-56.

32 Roberts JS, Connell CM: IIIness representations among first-degree relatives of people with Alzheimer disease. Alzheimer Dis Assoc Disord 2000; 14: 129-136.

33 Roberts JS, Cupples LA, Relkin NR, Whitehouse PJ, Green RC: Genetic risk assessment for adult children of people with Alzheimer's disease: the Risk Evaluation and Education for Alzheimer's Disease (REVEAL) Study. J Geriatr Psych Neur 2005; 18 : 250.

34 Marteau TM, Roberts S, LaRusse S, Green RC: Predictive genetic testing for Alzheimer's diseases: impact upon risk perception. Risk Anal 2005; 25: 397.

35 Hendrie HC, Albert MS, Butters MA et al: The NIH Cognitive and Emotional Health Project. Alzheimers Dement 2006; 2: 12-32.

36 Taylor SE: Adjustment to threatening events: a theory of cognitive adaptation. Am Psychol 1983; 38: 1161-1173.

37 Penziner $\mathrm{E}$, Williams JK, Erwin $\mathrm{C}$ et al: Perceptions of discrimination among persons who have undergone predictive testing for Huntington's disease. J Med Genet B Neuropsychiatr Genet 2008; 147: 320-325.

38 Zick CD, Mathews CJ, Roberts JS, Cook-Deegan R, Pokorski RJ, Green RC: Genetic testing for Alzheimer's disease and its impact on insurance purchasing behavior. Health Aff 2005; 24: 483-490.

39 Taylor DH, Cook-Deegan R, Hiraki S, Roberts JS, Blazer D, Green RC: Genetic testing for Alzheimer's and long-term care insurance. Health Aff 2010; 29: $102-108$.

40 Hallowell N, Ardern-Jones A, Eeles $\mathrm{R}$ et al: Communication about genetic testing in families of male BRCA1/2 carriers and non-carriers: patterns, priorities and problems. Clin Genet 2005; 67: 492-502.

41 Hughes C, Lerman C, Schwartz M et al: All in the family: evaluation of the process and content of sisters' communication about BRCA1 and BRCA2 genetic test results. Am J Med Genet 2002; 107: 143-150.

42 Clarke A, Richards M, Kerzin-Storrar L et al: Genetic professionals' reports of nondisclosure of genetic risk information within families. Eur J Hum Genet 2005; 13: $556-562$

\section{APPENDIX A \\ SURVEY ITEMS FOR IGT-AD DISTRESS SUBSCALE}

The questions below are about some specific responses you may have had after receiving your genetic test results. Indicate whether you have experienced each statement never, rarely, sometimes, or often in the past week.

In the past week, I have experienced...

Feeling upset about my test result

Feeling sad about my test result

Feeling anxious or nervous about my test result

Feeling a loss of control

Having problems enjoying life because of my test result

Worrying about my risk of getting AD

Being uncertain about what my test results mean about my risk of developing $A D$

Being uncertain about what my test result means for my children's and/or family's AD risk

Feeling frustrated that there are no definite AD prevention guidelines for me

Feeling concerned about how my test results will affect my insurance status

Having difficulty talking about my test results with family members

Feeling regret about getting my test results 